\title{
Pleural fluid due to papillary thyroid cancer
}

\author{
TOMOHIRO TAMURA ${ }^{1}$, TOSHIHIRO SHIOZAWA ${ }^{2}$, HIROAKI SATOH ${ }^{1}$, KOICHI KURISHIMA $^{1}$, \\ KATSUNORI KAGOHASHI ${ }^{1}$, NORIO TAKAYASHIKI ${ }^{3}$ and NOBUYUKI HIZAWA ${ }^{2}$ \\ ${ }^{1}$ Division of Respiratory Medicine, Mito Medical Center; ${ }^{2}$ Faculty of Medicine; ${ }^{3}$ Division of Pathology, \\ Mito Medical Center, University of Tsukuba, Ibaraki 310-0015, Japan
}

Received December 8, 2014; Accepted May 14, 2019

DOI: $10.3892 / \mathrm{ol} .2019 .10383$

\begin{abstract}
We report herein a rare case of massive pleural effusion caused by papillary thyroid cancer, which was accompanied by multiple pulmonary metastasis. A 91-year-old male patient presented with shortness of breath due to massive right pleural fluid. Cytological specimens, which were obtained from pleural fluid by thoracentesis, and was consistent with that observed in surgically resected thyroid cancer 6-year previously. Immunocytochemical staining of the cells was positive for cytokeratin (CK)-7, CK-19, and positive for thyroglobulin. Massive pleural fluid due to a metastatic from papillary thyroid cancer is very rare but may develop in long-term survivors with this disease as observed in this case.
\end{abstract}

\section{Introduction}

Thyroid cancer is not a rare cancer but has a relatively slow progression in many patients. However, it may metastasize to distant organs including lungs as disease progression. Lung metastases of thyroid cancer are those to the lung parenchyma, such metastases usually have multiple small nodules in both lungs $(1,2)$. On the contrary, however, pleural fluid due to thyroid cancer is a rare etiology, to our knowledge, and only 51 cases have been reported (3-19). Pleural fluid due to thyroid cancer has been reported to be less than $1 \%$ in all the patients with malignant pleural fluids $(4,11)$. Papillary thyroid cancer was the major histologic type of thyroid cancer with pleural effusion, and other histologic types were few (3). Thyroid cancer patients with pleural fluid, regardless of tissue type, were long-term survivors (3-19). In general, diagnosis of malignant fluid is established by positive pathological findings using specimens from pleural fluid (20). Thoracentesis using fine needle is usually used, but in some cases pleural biopsy or thoracoscope is performed if the appropriate materials cannot be obtained by thoracentesis (21). As for the therapy,

Correspondence to: Dr Hiroaki Satoh, Division of Respiratory Medicine, Mito Medical Center, University of Tsukuba, Miyamachi 3-2-7, Mito, Ibaraki 310-0015, Japan

E-mail: hirosato@md.tsukuba.ac.jp

Key words: pleural effusion, thyroid cancer drainage using thoracic tube is indicative for the patients with dyspnea, especially those with chemotherapy-resistant malignancies (22). As for therapy, pleurodesis is usually performed after pleural fluid drainage. However, as this is a rare condition, standard therapy has not yet established. This case report documents our clinical experience, we present herein a patient with pleural fluid due to papillary thyroid cancer, and reviewed previously reported such cases (3-19).

\section{Materials and methods}

The study was conducted in the Division of Respiratory Medicine, Mito Medical Center, University of Tsukuba and approved by the Ethics Committee of Division of Respiratory Medicine, Mito Medical Center, University of Tsukuba. An informed consent was obtained from the patient included in this study. Clinical evaluation of the lesions followed the guideline for diagnosis and treatment of the lung cancer (The Japan Lung Cancer Society 2018). All cytological specimens evaluated in our study underwent needle thoracic puncture under local anesthesia. Cytopathological examination was performed after standard hematoxylin and eosin staining of the specimens. Written informed consent was obtained from the patient for publication and this study was approved by the research ethics committee of Mito Medical Center, University of Tsukuba-Mito Kyodo General Hospital (vo. 1660). The patient was hospitalized from March 2013 to April 2013. Computed tomography used Aquilion 64 model manufactured by Toshiba Medical Systems (Tokyo, Japan). Cytological specimens obtained from pleural fluid were fixed in $10 \%$ buffered formalin, embedded in paraffin and stained with HE method routinely for histological examination. Immunostaining of cytokeratin 7 (CK-7), CK-19, and thyroglobulin was performed on thyroid cancer cells obtained from pleural fluid that were washed and fixed methanol before treatment with serum-free protein block (Code No. X0909; DAKO Tokyo, Japan). These cells were probed with antibodies to CK-7 (DAKO M7018; DAKO Tokyo, Japan; 1:300), CK-19 (DAKO M0888; DAKO Tokyo, Japan; 1:100), and thyroglobulin (Envision FLEX-Thyroglobulin, IR509; DAKO Tokyo, Japan; 1:200). Slides were incubated with the primary antibody for $30 \mathrm{~min}$ at room temperature. Immunoreactivity was detected using an indirect streptavidin-biotin method (LSAB kit; DAKO, Tokyo, Japan) and horseradish peroxidase. Bound antibody was detected as a brown stain. 
We performed a search on PubMed using the terms 'thyroid cancer', 'pleural fluid', and 'pleuritis' to identify similar cases, restricting the papers to those published in English. The articles detected are shown in References (Reference no. 3-19). Fifty-one patients of pleural fluid due to thyroid cancer were confirmed. Clinicopathological features of these patients were shown in Table I.

\section{Case report}

A 91-year-old male presented to the hospital with a 1-mo history of dyspnea on effort, which had worsened progressively over the previous few days. He had a 68-year history of tuberculaous pleuritis of the left and a 6-year history of surgically treated thyroid cancer. Physical examination showed that he was afebrile and had poor performance status. Neck examination showed well-healed cervical scars without adenopathy and chest examination showed dullness on the right side occupying one third of the chest. The peripheral blood test did not show leukocytosis and elevated levels of C-reactive protein, carcinoembryonic antigen, and CA19-9. A chest radiograph showed thickening and calcification of the left pleura, and small nodules in both lungs with pleural fluid in the right. Chest CT revealed massive right pleural fluid causing atelectasis of the right lung and shift of the mediastinum to the left side. Chest radiograph and chest CT scan also showed thickening and calcification of the left pleura, and well-circumscribed nodules measuring up to $15 \mathrm{~mm}$ in both lungs (Fig. 1). Primary or metastatic pulmonary cancer with pleural fluid, or recurrence of tuberculosis was highly suspected. Thoracentesis of pleural fluid was performed to alleviate severe dyspnea, yielding a serous fluid with malignant cells on cytological examination. Cancer cells, which had intra-nuclear inclusion body, were found in pleural fluid, and immunocytochemical staining of the cells was positive for cytokeratin (CK)-7, CK-19, and positive for thyroglobulin (Fig. 2). The diagnosis of massive pleural fluid due to metastatic papillary thyroid cancer was established. The patient's poor general condition and refusal prevented us from further treatment. Thereafter, the patient followed up with palliative care and died of the disease one month after the diagnosis of pleural fluid due to thyroid cancer. Diagnosis of pleural fluid due to papillary thyroid cancer was confirmed at autopsy. Informed patient consent was obtained from the family of the patient.

\section{Discussion}

Massive pleural fluid caused by thyroid cancer is very rare. To our best knowledge, there have been reported only 51 cases (3-19). According to a review by Vassilopoulou-Sellin and Sneige at the MD Anderson Cancer Center, 10 (0.6\%) had malignant pleural fluid that developed during the course of the disease among 1772 differentiated papillary thyroid cancer patients (4). In a recent review by Olson $e t$ al at the Johns Hopkins Hospital, only 6 patients with pleural fluid resulting from metastasis of thyroid primaries over the last 26 years, which comprised $0.67 \%$ of all malignant pleural fluids (11). Most of the 21 patients had papillary thyroid cancer except for a few cases with both papillary and undifferentiated carcinomas (19), follicular thyroid cancer (16) and Hürthle cell

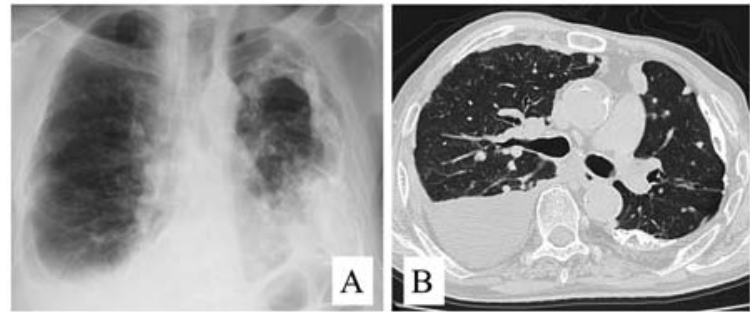

Figure 1. Chest radiograph (A) and chest CT scan (B) showed massive pleural fluid in the right, thickening and calcification of the left pleura, and well-circumscribed nodules measuring up to $15 \mathrm{~mm}$ in both lungs.

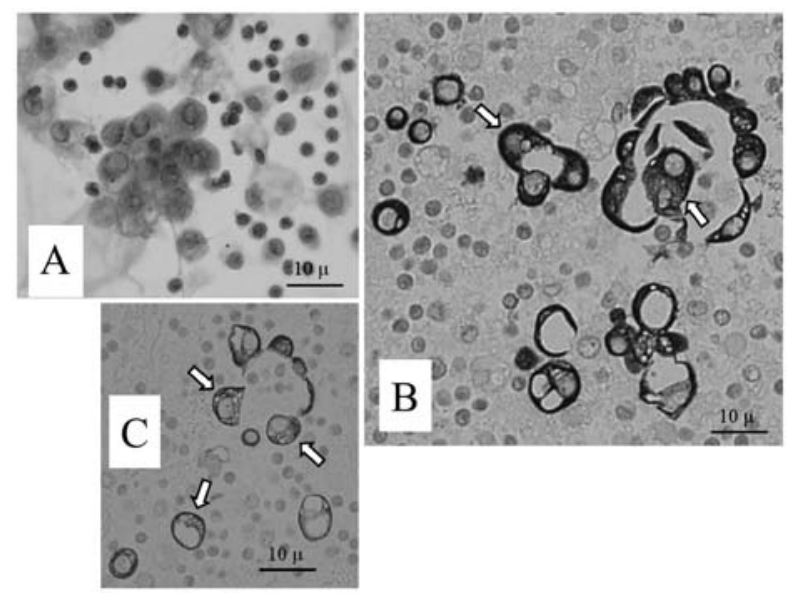

Figure 2. Cell pellet sample obtained by needle aspiration showed cancer cells with intranuclear inclusion body (Papanicoloau staining) (A, 400x), and immunocy tochemical staining of the cells was positive for cytokeratin (CK)-7 (B, 400x), CK-19, and positive for thyroglobulin (C, 400x).

thyroid cancer (9), which is usually classified with a certain type of follicular thyroid cancer (9). In our case, we compared the pathological findings of the specimens obtained from pleural fluids and those surgically resected specimens, which were 6-year previously in our hospital. They were consistent and confirm the diagnosis of papillary thyroid cancer. Vassilopoulou-Sellin and Sneige reported that pleural fluid appeared 61 to 132 months after the initial diagnosis of thyroid cancer in 4 patients (4). Together with the result of our case, these facts imply that pleural fluid may develop in long-term survivors with this disease.

With regards to distant metastases, Vassilopoulou-Sellin and Sneige reported that all their 10 patients had radiologically apparent lung metastases at the time pleural fluid was found (4). Other reports also pointed out lung metastases $(3,5,7,12-14,17)$. Vernon et al reported a patient with a left iliac crest metastasis and multiple bilateral pulmonary metastases were also found (5). On the other hand, only one of the 6 patients had bone metastases, but others had no pathological evidence of distant metastasis in their institutional review by Olson et al (11). Our patient had multiple bilateral pulmonary metastases, which had been detected a few months prior to the pleural fluid.

The diagnosis of massive pleural fluid in our patient was not difficult with pleural immunocytochemical findings. In general, however, it may be difficult because of its rarity of pleural dissemination from thyroid cancer and the similarity of the symptoms and signs to those of advanced chronic 


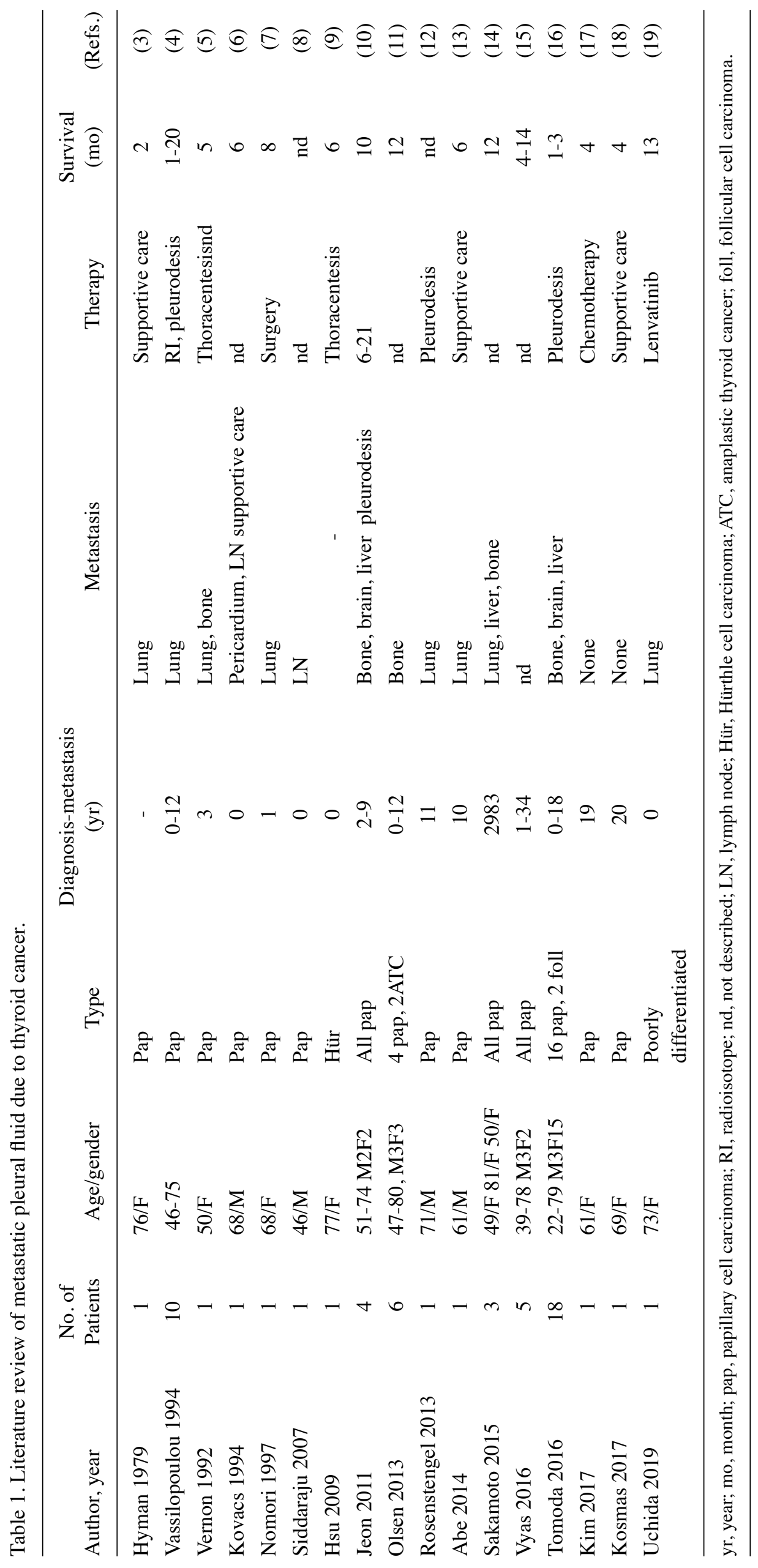


pulmonary diseases. Negative cytological results of pleural fluid might be required in evaluation of differential diagnosis of other causes of pleural fluid. Our patient had a history of tuberculous pleuritis, therefore, we had to include it as a differential diagnosis.

As for the therapy, in general, in patients with pleural fluid due to chemo-sensitive cancers, systemic chemotherapy is indicative for them (23-25). In those with chemo-insensitive cancers, on the other hand, chest tube drainage and pleurodesis are treatment choices for them (23-25). Vassilopoulou-Sellin and Sneige reported that pleural fluids in their patients were treated with local radioisotopes or sclerosing agents, systemic radioiodine or chemotherapy, or both (4). They also reported that appearance of pleural fluid preceded death by 1 to 20 months (median, 11 months) and pleural fluid was associated with greatly shortened survival time in all cases (3,4,7,9-11,14-19). In our patient, chest tube drainage and pleurodesis were considered, but only thoracentesis was performed because of his contra-lateral calcified pleural thickening and poor respiratory condition and died of thyroid cancer one month after the diagnosis of pleural fluid.

In summary, pleural metastasis from papillary thyroid cancer is a potential cause of massive pleural fluid, although very rare. The current case extended the clinical spectrum of metastatic potential to the pleura of papillary thyroid cancer. As for the therapy, long-term palliation can be the treatment choice for these patients as there has not established any standard therapy for them.

\section{Acknowledgements}

The authors would like to thank Mr Jyunichi Hakamtsuka (Division of Central Clinical Laboratory, Mito Medical Center, University of Tsukuba) for his technical assistance with cytological analysis.

\section{Funding}

No funding was received

\section{Availability of data and materials}

The datasets during and/or analyzed during the current study available from the corresponding author on reasonable request.

\section{Authors' contributions}

TT, TS, HS, KKa were responsible for the design of the study and interpretation of the data. They also have revised critically the manuscript for important intellectual content. HS, KKa, $\mathrm{NT}, \mathrm{NH}$ were responsible for the data acquisition, selection and analysis and clinical interpretation of the data. TT, HS, $\mathrm{KKu}, \mathrm{NT}$ were responsible for the data analysis and interpretation. All authors contributed to the writing of the manuscript. All authors read and approved the final version of manuscript.

\section{Ethics approval and consent to participate}

The study was conducted in the Division of Respiratory Medicine, Mito Medical Center, University of Tsukuba and approved by the Ethics Committee of Division of Respiratory Medicine, Mito Medical Center, University of Tsukuba. An informed consent was obtained from the patient included in this study.

\section{Patient consent for publication}

Informed consent for publication was obtained from the family of the patient.

\section{Competing interests}

The authors declare that they have no competing interests.

\section{References}

1. Quint LE, Park CH and Iannettoni MD: Solitary pulmonary nodules in patients with extrapulmonary neoplasms. Radiology 217: 257-261, 2000.

2. Massin JP, Savoie JC, Gamier H, Guiraudon C, Leger FA and Bacourt F: Pulmonary metastases in differentiated thyroid carcinoma. Study of 58 cases with implications for the primary tumor treatment. Cancer 53: 982-992, 1984.

3. Hyman MP: Papillary and undifferentiated thyroid carcinoma presenting as a metastatic papillary serous effusion. A case report. Acta Cytol 23: 483-486, 1979.

4. Vassilopoulou-Sellin R and Sneige N: Pleural effusion in patients with differentiated papillary thyroid cancer. South Med J 87: 1111-1116, 1994.

5. Vernon AN, Sheeler LR, Biscotti CV and Stoller JK: Pleural effusion resulting from metastatic papillary carcinoma of the thyroid. Chest 101: 1448-1450, 1992.

6. Kovacs CS, Nguyen GK, Mullen JC and Crockford PM: Cardiac tamponade as the initial presentation of papillary thyroid carcinoma. Can J Cardiol 10: 279-281, 1994.

7. Nomori H, Horio H, Mimura T and Morinaga S: Massive hemoptysis from an endobronchial metastasis of thyroid papillary carcinoma. Thorac Cardiovasc Surg 45: 205-207, 1997.

8. Siddaraju N, Viswanathan VK, Saka VK, Basu D and Shanmugham C: Fine needle aspiration of follicular variant of papillary thyroid carcinoma presenting with pleural effusion: A case report. Acta Cytol 51: 911-915, 2007.

9. Hsu KF, Hsieh CB, Duh QY, Chien CF, Li HS and Shih ML: Hürthle cell carcinoma of the thyroid with contralateral malignant pleural effusion. Onkologie 32: 47-49, 2009.

10. Jeon MJ, Yim JH, Kim EY, Kim WG, Kim TY, Kim WB and Shong YK: Four cases of malignant pleural effusion in patients with papillary thyroid carcinoma. Endocrinol Metab 26: 330-334, 2011.

11. Olson MT, Nuransoy A and Ali SZ: Malignant pleural effusion resulting from metastasis of thyroid primaries: A cytomorphological analysis. Acta Cytol 57: 177-183, 2013.

12. Rosenstengel A, Lim EM, Millward M and Lee YG: A distinctive colour associated with high iodine content in malignant pleural effusion from metastatic papillary thyroid cancer: A case report. J Med Case Rep 7: 147, 2013.

13. Abe T, Suzuki M, Shimizu K, Shinagawa N, Oizumi S, Matsuno Y, Miyazaki M, Tanino M, Tanaka S and Nishimura M: Anaplastic transformation of papillary thyroid carcinoma in multiple lung metastases presenting with a malignant pleural effusion: A case report. J Med Case Rep 8: 460, 2014.

14. Sakamoto RI, Sumida LC, Lum CA and Tauchi-Nishi PS: Recurrent papillary thyroid carcinoma with pleural metastasis diagnosed by effusion cytology: A report of cases with clinicopathologic correlation. Hawaii J Med Public Health 74: 51-56, 2015.

15. Vyas $\mathbf{M}$ and Harigopal M: Metastatic thyroid carcinoma presenting as malignant pleural effusion: A cytologic review of 5 cases. Diagn Cytopathol. 44: 1085-1089, 2016.

16. Tomoda C, Ogimi Y, Saito F, Masaki C, Akaishi J, Matsuzu K, Suzuki A, Uruno T, Ohkuwa K, Shibuya H, et al: Outcome and characteristics of patients with malignant pleural effusion from differentiated thyroid carcinoma. Endocr J 63: 257-261, 2016. 
17. Kim H, Park YW, Oh YH, Sim J, Ro JY and Pyo JY: Anaplastic transformation of papillary thyroid carcinoma only seen in pleural metastasis: A case report with review of the literature. Head Neck Pathol 11: 162-167, 2017.

18. Kosmas K, Tsonou A, Mitropoulou G, Salemi E, Kazi D and Theofanopoulou A: Malignant pleural effusion from papillary thyroid carcinoma diagnosed by pleural effusion cytology: A case report. Diagn Cytopathol 46: 204-207, 2018.

19. Uchida T, Yamaguchi H, Nagamine K, Yonekawa T, Nakamura E, Shibata N, Kawano F, Asada Y and Nakazato M: Rapid pleural effusion after discontinuation of lenvatinib in a patient with pleural metastasis from thyroid cancer. Endocrinol Diabetes Metab Case Rep 2019: EDM180158, 2019.

20. Heffner JE: Diagnosis and management of malignant pleural effusions. Respirology 13: 5-20, 2008.
21. Casal RF, Eapen GA, Morice RC and Jimenez CA: Medical thoracoscopy. Curr Opin Pulm Med 15: 313-320, 2009.

22. Kastelik JA: Management of malignant pleural effusion. Lung 191: 165-175, 2013.

23. Parwani AV, Chan TY and Ali SZ: Significance of psammoma bodies in serous cavity fluid: A cytopathologic analysis. Cancer 102: 87-91, 2004.

24. Zahid I, Routledge T, Billè A and Scarci M: What is the best treatment for malignant pleural effusions? Interact Cardiovasc Thorac Surg 12: 818-823, 2011.

25. Rodriguez-Panadero F and Romero-Romero B: Management of malignant pleural effusions. Curr Opin Pulm Med 17: 269-273, 2011. 